\title{
Révolution de soi, révélation de l'autre : figures du transport dans la poésie de Lotte Kramer
}

\section{Nathalie Vincent-Arnaud}

\section{(2) OpenEdition}

1 Journals

Édition électronique

URL : http://journals.openedition.org/esa/3379

DOI : 10.4000/esa.3379

ISSN : 2650-2623

\section{Éditeur}

Société de stylistique anglaise

\section{Édition imprimée}

Date de publication : 31 décembre 2018

Pagination : 161-172

ISBN : 978-2-36442-083-0

ISSN : 2116-1747

\section{Référence électronique}

Nathalie Vincent-Arnaud, «Révolution de soi, révélation de l'autre : figures du transport dans la poésie de Lotte Kramer », Études de stylistique anglaise [En ligne], 13 | 2018, mis en ligne le 20 mars 2019, consulté le 20 mars 2020. URL : http://journals.openedition.org/esa/3379 ; DOI : https://doi.org/ 10.4000/esa.3379 


\title{
Révolution de soi, révélation de l'autre : figures du transport dans la poésie de Lotte Kramer
}

\author{
Nathalie VINCENT-ARNAUD \\ Université Toulouse - Jean Jaurès
}

Au-delà de l'exercice de style qui semble souvent un peu contraint mais qui est une de ces petites tentations paratextuelles auxquelles on cède presque toujours, la paronomase est ce jeu (au sens d'entre-deux), cet espace ouvert entre deux notions qu'elle permet de travailler, de creuser, poussant plus loin les résonances de celles-ci dans notre "bouchoreille " toujours en éveil. À travers ce qui, au-delà de la simple symétrie de surface, semble être une relation de cause à effet, le titre ci-dessus réunit deux notions - révolution et révélation - fondamentales pour appréhender l'œuvre poétique de Lotte Kramer dans ce qu'elle a de plus intime, de plus texturé (adjectif dérivé de ce que Jean-Michel Adam a nommé "faits de texture ", définis comme des "phénomènes linguistiques identifiables à un niveau micro-structurel" $[1994,19])$. Si, dans ce titre comme partout ailleurs, le terme de révolution dit une forme de violence et de bouleversement brusque d'une structure, il n'en annonce pas moins toujours, en premier lieu, un parcours autour d'un axe central qui ramène l'objet concerné au même point (définition qui n'est donc autre que le substrat spatial de la métaphore fournissant l'acception la plus courante). Que ce parcours s'accompagne d'une révélation, ou que cette dernière en

Forgé par Paul Valéry, ce terme est repris par Jean-Michel Maulpoix : "Il y a, en poésie, une certaine façon d'aller vers le sens à travers la matière sonore de la langue, en se laissant conduire par elle, en écrivant à l'oreille, dans le 'bouchoreille du poème' " (2013, 43-44). Les italiques sont de l'auteur. 
RÉVOLUTION DE SOI, RÉVÉLATION DE L'AUTRE :

FIGURES DU TRANSPORT DANS LA POÉSIE DE LOTTE KRAMER

soit l'aboutissement n'a pas de quoi étonner lorsque le cheminement concerné présente une certaine intensité de rencontres, d'instants de vie, de chocs esthétiques, de heurts répétés de la subjectivité avec le monde qui l'entoure. Ces deux notions étroitement corrélées sont réunies à travers celle du transport qui fournit sans aucun doute le socle métaphorique de la poésie de Lotte Kramer. C'est à l'origine même de l'itinéraire existentiel de l'auteur qu'on rencontre la première actualisation de ce terme : en effet, c'est par le célèbre Kindertransport (ou Children's Transport), que Lotte Kramer, allemande et juive, née à Mayence en 1923, est arrivée en Angleterre en 1939 à l'âge de 15 ans, pays qu'elle n'a jamais plus quitté et dont elle a adopté la langue, dans sa vie comme dans l'écriture qu'elle pratique depuis la fin des années soixante.

Survenue tardivement dans une vie d'abord occupée à la peinture (première forme d'art pratiquée par l'auteur), cette écriture est née du silence et de la solitude, de la confrontation avec soi-même et avec ses souvenirs dont on a soudain ouvert la boîte de Pandore comme le révèle la préface autographe de New and Collected Poems, volume qui réunit l'ensemble des recueils précédents :

In 1968 we moved from the London area to Peterborough because of my husband's new job. [...] I felt isolated. People were friendly but I was on my own quite a lot as my husband's work took him abroad very often and my son was in Oxford. It sent me back to the early years, when I was transported to England from a close family unit and I was an alien when war broke out.

Memories of childhood in Nazi Germany, which I had buried for many years, came back and insisted on being written about. $(2011,7)$

Étrange agentivité que celle de ces souvenirs qui, non contents de se frayer un passage à travers la conscience, réclament leur inscription, deviennent cette "voix qui insiste" qui est déjà, selon l'écrivain Marie Darrieussecq (2000), une manière de définition du style, du flux qui informe et sculpte la matière de la langue. L'image singulière de cette insistance des souvenirs et de leur impatience à se déverser sur la page se fait d'ailleurs résurgence à l'orée du tout premier recueil publié par l'auteur, Ice-Break; le poème y apparaît en effet mu par une force autonome, une nécessité dont rien ne peut venir enrayer le déroulement imperturbable : «[...] the poem insists on its flow / With the ice-break of words " (Kramer 2011, 25). 


\section{Clés mémorielles}

Comme le suggèrent les précisions biographiques données par l'auteur dans l'extrait de préface déjà mentionné, la figure de Lotte Kramer ainsi que la place qu'elle occupe dans la poésie britannique contemporaine font résonner toute une partie de l'Histoire collective du $\mathrm{XX}^{\mathrm{e}}$ siècle dans laquelle s'enracine un parcours individuel affirmant sa singularité au travers d'une poésie dont l'auteur déclare sans ambages (toujours dans la préface déjà mentionnée) : «Poetry became my life " (Kramer 2011，7). L'écriture pour perpétuer la vie, tout en en exhumant simultanément les origines : voilà qui n'a peut-être pas tout à fait un caractère de nouveauté, mais qui n'en acquiert pas moins une résonance particulière chez un individu que rien de particulier, jusqu'alors, n'avait disposé à être auteur, si ce n'est, assurément, l'amour de la poésie mais aussi la fascination éprouvée pour l'écriture - au double sens de graphie et d'activité créatrice - d'un père disparu évoqué à deux reprises, au fil des recueils, comme "a minor Proust" (Kramer 2005, 15) et "a witty playwright " (Kramer 2009, 20).

La venue à l'écriture de Lotte Kramer s'est faite par effraction subite du silence d'une vie d'adulte en Angleterre suivant, comme tant d'autres, un cours ordinaire, ordonné et placide. C'est cette effraction que dit, dans l'intitulé déjà mentionné, l'image du mot brise-glace ("the icebreak of words "), à laquelle fait pleinement écho un intitulé bien plus tardif, celui du dernier recueil en date, Turning the Key. Si, dans ce dernier titre, le mode d'entrée dans l'espace mémoriel semble s'être considérablement adouci, la forme en -ing n'en opère pas moins une saisie en flagrant délit d'un processus continu, d'une exploration au long cours, de la pénétration, par une porte dérobée dans la façade lisse du présent, dans un univers dont la première étape se nomme, de manière éloquente, "Identity" :

A river, wandering

Through a bed of rocks

Never quite homing in one place,

Tasting the difference

Of earth's

Textures, lushness

And dryness for my

Long journey $[\ldots]$

(Kramer 2009, 9) 
RÉVOLUTION DE SOI, RÉVÉLATION DE L'AUTRE :

FIGURES DU TRANSPORT DANS LA POÉSIE DE LOTTE KRAMER

Passage en force par le brise-glace, en douceur par la clé que l'on tourne ou par la rivière sinueuse dont on suit le cours au fil d'aspérités et de paysages variés, le franchissement de la frontière s'obstine au fil des ans, des décennies, jusqu'à se faire modalité d'écriture et, chemin faisant, découverte singulière par l'auteur de ce processus logé au cœur de tout acte scriptural tel que l'a évoqué Orhan Pamuk :

Pour moi, être écrivain, c'est découvrir patiemment, au fil des années, la seconde personne, cachée, qui vit en nous, et un monde qui sécrète notre seconde vie : l'écriture m'évoque en premier lieu [...] l'homme qui, enfermé dans une chambre, se replie sur lui-même, seul avec les mots, et jette, ce faisant, les fondations d'un nouveau monde. [...] Écrire, c'est traduire en mots ce regard intérieur, passer à l'intérieur de soi [...]. (Pamuk 2006)

C'est cette plongée subreptice dans ce monde inconnu de la "seconde vie " que traduit aussi le titre du recueil Black over Red. La toile saturée de couleur de Rothko - présente en première de couverture - y augure les surfaces à creuser, la dualité, l'entreprise permanente de déchiffrement et d'excavation, mais aussi les couleurs mêlées d'une épaisseur existentielle dont la texture riche et moirée suscite tout à la fois tension et tentation comme en témoigne le poème éponyme du recueil :

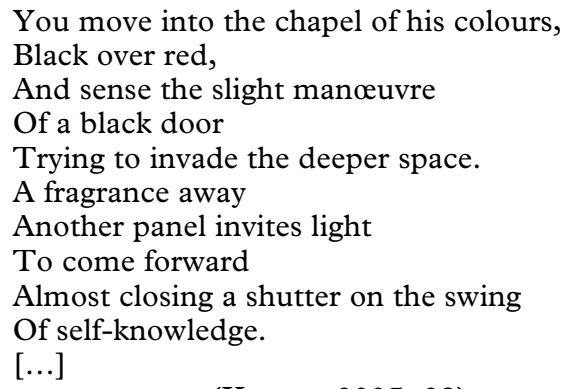

(Kramer 2005, 28)

De la figura du poème (sa présentation sur la page), alternance d'élans et de rétractions, au choc répété des antonymes et des paradoxes, en passant par la lumière qui s'infiltre et cède brutalement la place au volet qui se rabat, tout semble, dans ce poème, rappeler les atermoiements et la tentation du silence évoqués par George Steiner $(1967,7)$ à propos de ceux que l'on appelle les "écrivains de l'Holocauste " (étiquette très floue également assignée à Lotte Kramer en Angleterre et qui, de toute évidence, ne suffit pas telle quelle à rendre justice à la singularité de son écriture). Mais chez elle, immanquablement, la voix parvient à percer la 
toile du silence, à recueillir et à faire s'épanouir ces "fleurs du silence " diffusant leur parfum ("A fragrance away ") jusqu'à ouvrir l'horizon d'une révélation, d'un instant qui bascule vers la densité d'une perception (" on the swing / Of self-knowledge "). Corrélativement, les apparitions, rares mais régulières, de haïkus - ainsi intitulés $-{ }^{3}$ au fil des recueils s'éprouvent comme autant de fulgurances qui, par leur brièveté et leur saturation sémantique, figurent des jalons, des passages, des seuils entre le dire et son absence, entre l'instant créateur et le chatoiement du silence qui l'a engendré.

\section{Apparitions poétiques}

Comme Lotte Kramer le souligne dans la préface d'un de ses recueils, la poésie est le seul genre littéraire qu'elle ait jamais pratiqué. En guise d'explication au moins partielle, elle mentionne tour à tour son inclination pour la poésie allemande étudiée assidûment dans son enfance et pour la poésie de langue anglaise découverte plus tard, par ses lectures mais aussi par l'écoute assidue d'une émission radiophonique consacrée à Emily Dickinson : "I began to read English poetry, heard a programme on Emily Dickinson on the BBC and that was my start. I began to write seriously and memories came flooding in " (Kramer 2011, 7).

$\mathrm{Ce}$ flot continu et insistant qui fait résurgence de manière systématique à travers la forme poétique nous convie à examiner les spécificités de celle-ci à travers tout d'abord son empreinte la plus matérielle. Comme le fait observer Jean-Michel Maulpoix, le poème "fait apparition ", il isole, il distingue, il singularise, et au sein de ce splendide isolement sur la page il creuse l'image issue de la mémoire :

[...] si le poème est un objet verbal où la langue même prend voix, c'est aussi un texte qui existe spatialement, typographiquement. [...] Il existe pour l'œil avant d'exister pour l'oreille. Il segmente et espace singulièrement le langage sur la page. Il fait apparition. (Maulpoix 2013, 73)

La formule provient du proverbe japonais : «Les mots qu'on n'a pas dits sont les fleurs du silence" .

3 On note six haïkus (dont trois formant une séquence) distribués tout au long du volume New and Collected Poems. 
RÉVOLUTION DE SOI, RÉVÉLATION DE L'AUTRE :

FIGURES DU TRANSPORT DANS LA POÉSIE DE LOTTE KRAMER

C'est à travers cette série d'"apparitions " que se dévoilent et prennent corps sur la page le processus d'anamnèse ainsi que la distillation des souvenirs et des états qu'ils suscitent dans le présent de l'énonciation. Au fil de cette quête du "lointain intérieur " par la poésie, au sein de la forme minuscule se multiplient les arrêts sur images, la fascination pour les vies minuscules rencontrées en Allemagne, en Angleterre ou ailleurs. Ces vies entr'aperçues ou côtoyées de près sont ainsi reconstruites par une mémoire insistante en quête de surfaces à percer, d'espaces à pénétrer, d'effractions à commettre pour retrouver des voix, des silhouettes, des objets aux devenirs incertains. En témoignent les perhaps, must have been et autres emplois spéculatifs de would qui viennent régulièrement trahir le doute quant à un invisible ou une absence auxquels l'imaginaire tente de donner corps (les disparus, les déportés, les ombres vulnérables, les contours instables et fuyants qui rôdent dans le souvenir) :

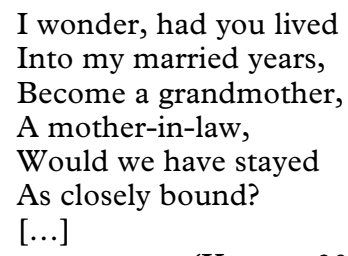

(Kramer 2011, 239)

C'est, chez Lotte Kramer, la métaphore du passage, du voyage à rebours qui construit l'imaginaire en déroulant un paysage peuplé de formes, de personnages multiples, de seuils à franchir. Se coulant parmi les miniatures allégoriques qui jalonnent l'œuvre en y insinuant des images répétées de rivières et de traversées (de "River Incantation " et "My Three Rivers " à "Crossing a Bridge »), le fleuve de l'enfance, le Rhin, s'y fait emblème d'un territoire identitaire brouillé et hybride, entre stable et instable, solide et mouvant. Ses apparitions répétées au fil des poèmes et surtout l'imaginaire qui en découle servent d'ancrage pour une appréhension du monde et du langage qui le dit; ainsi surgit, à travers le poème "Bilingual ", la cartographie détaillée d'un entre-deux identitaire, d'un moi étranger dans chacune des deux langues explorées, errant aux confins des origines et des destinations tout en jouissant de son étonnement et d'une forme d'émerveillement qui perdure :

When you speak German

The Rhineland opens its watery gates,

Lets in strong currents of thought. 
Sentences sit on shores teeming

With certainties. You cross bridges

To travel many lifetimes

Of a captive's continent.

When you speak English

The hesitant earth softens your vowels.

The sea - never far away - explores

Your words with liquid memory.

$[\ldots]$

(Kramer 2011, 137)

Cette esthétique du surgissement et de la segmentation, bien que forgée par à-coups, n'en construit pas moins une cohérence. Voix et visages de femmes et d'enfants jaillissent ainsi de l'ombre au détour des bifurcations de la mémoire, s'immisçant dans le flot du recueil par le biais de leurs monologues respectifs - le discours direct rapporté fournissant alors l'armature du poème - ou de portraits dont la tonalité peut à l'occasion se faire lyrique; il en est ainsi, dans "Ode to M.F.", de l'évocation de la femme qui a recueilli l'enfance naufragée et qui, par sa chaleur, son chant, sa lecture de Dickens, s'impose comme l'un des viatiques du voyage mémoriel :

She met us in a grim-grey station

And warmth spilled from our eyes,

A light on that smoked-filled morning

Surrounded and spread from her side.

Her Irish voice was melodious,

Her exuberance infected us all,

She could love and hate profoundly

And her temperament held us in thrall.

[...]

(Kramer 2009, 17)

À l'instar de cette oscillation entre voix étrangères et replis vers l'intériorité qui s'en nourrit, les occurrences alternées des formes du présent et du passé, mais aussi du $I$, du we, du you, d'une troisième personne explicitement nommée (père, mère, parent, ami) ou demeurant dans l'obscurité de l'implicite, font des recueils un long couloir qu'arpentent tour à tour des figures solitaires, fragments détachés du passé, où défilent des paysages chargés d'allégories, où le moi poétique déambule dans tous ses états. La convocation répétée de l'enfance tout au long de ces assauts de visions fugitives sert à évaluer la rémanence, la persistance d'un trauma que l'adulte est à même de s'approprier, de 
RÉVOLUTION DE SOI, RÉVÉLATION DE L'AUTRE :

FIGURES DU TRANSPORT DANS LA POÉSIE DE LOTTE KRAMER

qualifier, de transformer en "phrasés du vivre" (Macé 2016) qui construisent peu à peu sa singularité. C'est ainsi que peut s'appréhender la révolution de soi que fait advenir l'écriture, définie par Luc Lang comme une "manière de reconstruire, par le détour des mots, qui seuls font sens pour nous, une cohérence qui rende simplement acceptable et supportable cette réalité définitivement étrangère dans laquelle nous sommes précipités " (Lang 2011,36).

\section{Voix résurgentes}

Les traductions auxquelles Lotte Kramer se livre avec la constance d'une obsession d'un recueil à l'autre témoignent de retrouvailles régulières et intimes avec l'allemand et avec la poésie de l'enfance, du désir de se faire passeur de ce terreau premier, de tout un ensemble de mythes fondateurs de goûts, d'aspirations, d'élans et de réticences. La traduction intervient en point d'orgue de la geste poétique de l'auteur comme mémoire des formes, comme perpétuation de tout un univers mental, sensible, de récits et d'images, d'enchantements originels : à Lotte Kramer écrivain se mêle ainsi une seconde figure, celle de Lotte Kramer traductrice, qui s'érige dans la continuité de la précédente comme en témoigne la présence systématique, à la fin de ses recueils, de ses traductions de poèmes d'auteurs allemands de prédilection - Rilke, Hölderlin, Trakl, Hesse - semblant se mêler aux "voix chères qui se sont tues" et qui hantent l'ensemble de l'œuvre. La démarche de l'écrivain accueillant le flot des images du passé pour les articuler en une forme intelligible procède d'une seule et même opération mentale que celle de la traductrice, qui consiste à déchiffrer un discours en lui donnant sa place dans une langue d'accueil. L'exhumation et le transport des voix constituent, dans les deux cas, le principal enjeu, dont l'auteur-traductrice d'impressions et d'idiomes ne se départit jamais au fil d'une double trajectoire devenue une constante dans son œuvre. Celle-ci est sans doute illustrée en premier lieu par le passage de la peinture à la poésie dont elle a fait l'expérience soudaine et inattendue et qui semble signer l'avènement d'une perspective intersémiotique, une nouvelle frontière étant franchie pour permettre au langage verbal de prendre son essor à travers le surgissement impérieux déjà évoqué.

L'examen des poèmes traduits par Lotte Kramer révèle une nette domination de certains thèmes tels que l'exil, le déracinement, l'errance ou 
l'oppression : autant de thèmes largement explorés par la poésie allemande dans la mouvance quasi inextinguible du Romantisme qui lui est cher, autant de ponts jetés avec les stigmates de l'enfance allemande transplantée puis avec l'adolescence britannique emplie des Lieder de Schubert tout à la fois salvateurs et exhausteurs de la perte originelle (Kramer 2009, 17). La traduction apparaît ainsi comme le double, le Doppelgänger de l'écriture, une manière de révélation ultime de l'autre en soi, en écho à la devise que Carlos Batista, dans son Bréviaire $d u$ traducteur, attribue à un traducteur qui serait par essence schizophrène : "Traduire, c'est écrire accompagné " (2003, 14). Cet accompagnement, vecteur de réassurance du moi à travers la convocation simultanée du passé et du présent, est ici assuré par les figures tutélaires que font resurgir les ultimes arrêts sur images des différents recueils. Celles-ci rejoignent la cohorte des Mahler, Rothko, Nolde, Munch, Rilke et autres Caspar David Friedrich au panthéon des émotions artistiques - musicales, picturales, poétiques - égrenées au fil des textes comme stations successives du moi, carrefours polyphoniques, chant perpétuel d'une terre foisonnante devenue "langue-pays intérieur» (Gansel 2012, 91) arpenté tour à tour avec curiosité, appréhension et ferveur.

\section{Conclusion}

Entre texte original et texte traduit, enfouissement et renaissance, les figures du transport se mêlent dans l'œuvre de Lotte Kramer pour révéler une parole qui est giration inlassable sur soi et dans l'entour de soi. En témoignent les résurgences de voix, de silhouettes, d'objets et de couleurs dont s'abreuve cette parole, le passé venant peu à peu, au fil des poèmes et des recueils, se fondre à la toile pleinement habitée, pleinement investie, du présent poétique. Tel ce "cowslip ", cette primevère éclat de lumière qui surgit au détour d'un poème (Kramer 2005, 60) et dont l'auteur prend soin de nous préciser en note que son équivalent allemand est "Schlüsselblume" ("key flower»), chaque parcelle du temps retrouvé devient ce "délicat sismographe au cœur du temps "(Gansel 2012, 43) parcouru des frémissements moirés de l'intime, habité de cette tension permanente que traduit le dessin vibratile du poème en un jeu d'apparition/disparition d'une page à l'autre. 
"Il appartient au poète de dire, et peut-être toujours de chanter, ou de chercher ce qui reste chantable ${ }^{4}$, affirme Jean-Michel Maulpoix, reprenant les mots célèbres de Paul Celan (Maulpoix 2013, 101), et c'est bien ce chant retrouvé qui, chez Lotte Kramer, ouvre l'espace poétique : "A song / Of searching voices / For the many and the one" (Kramer 2009, 9). Clé des voix intérieures, des chants de la mémoire, guide à travers les champs de ruines du passé, l'œuvre poétique de Lotte Kramer s'impose comme parcours de re-connaissance de ce qui fait, étape après étape, page après page, cet assemblage par essence instable, toujours en révolution, toujours en quête de lui-même par-delà les ancrages successifs dans le temps et dans l'espace, qu'on appelle identité.

\footnotetext{
4 Les italiques sont de l'auteur.
} 


\section{BIBLIOGRAPHIE}

ADAM, Jean-Michel. 1994. "Style et fait de style : un exemple rimbaldien ». In P. CAHNÉ et G. MOLINIÉ (éd.), Qu'est-ce que le style ? Paris : Presses Universitaires de France, p. 15-43.

BATISTA, Carlos. 2003. Bréviaire du traducteur. Paris : Arléa.

DARRIEUSSECQ, Marie. 2000. «Qu'est-ce que le style ? », conférence donnée le 30 décembre 2000 dans le cadre de l'Université de tous les savoirs, $<$ http://www.canalu.tv/themes/sciences_humaines_sociales_de_1_education_et_de_1_inform ation/sciences_de_1_homme/philosophie/qu_est_ce_que_le_style>, consulté le 28 mai 2018.

GANSEL, Mireille. 2012. Traduire comme transhumer. Rennes : Calligrammes.

KRAMER, Lotte. 2005. Black over Red. Ware: Rockingham Press.

KRAMER, Lotte. 2009. Turning the Key. Ware: Rockingham Press.

KRAMER, Lotte. 2011. New and Collected Poems. Ware: Rockingham Press.

LANG, Luc. 2011. Délit de fiction. La littérature, pourquoi? Paris: Folio Essais.

MACÉ, Marielle. 2016. Styles : critique de nos formes de vie. Paris : Gallimard.

MAULPOIX, Jean-Michel. 2013. La Musique inconnue. Paris : Corti.

PAMUK, Orhan, 2006. " La valise de mon père », https://www.lemonde.fr/livres/article/2006/12/14/orhan-pamuk-la-valisede-mon-pere 845646_3260.html, consulté le 17 mai 2018. 
RÉVOLUTION DE SOI, RÉVÉLATION DE L'AUTRE :

FIGURES DU TRANSPORT DANS LA POÉSIE DE LOTTE KRAMER

STEINER, George. 1967. Language and Silence: Essays on Language, Literature, and the Inhuman. New York: Atheneum.

VINCENT-ARNAUD, Nathalie. 2018. « La poésie "brise-glace" de Lotte

Kramer (1923-). Trois poèmes traduits ». In La Main de Thôt $\mathrm{n}^{\circ} 5$, « Traduire la poésie ».

http://revues.univ-tlse2.fr/lamaindethot/index.php?id=708, consulté le 20 juin 2018. 